\title{
NEONATAL NURSING WORKLOAD - CAN IT BE MEASURED?
}

\author{
JM van den Heever
}

\section{ABSTRACT}

Contuning pressine on tertions

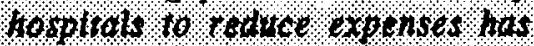

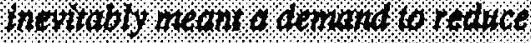

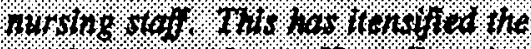

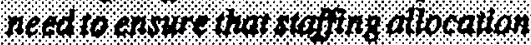

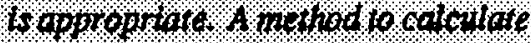

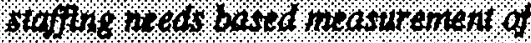

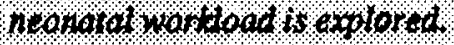

\section{INTRODUCTION}

In a political and socio-economic climate where ethical dilemmas of just distribution of scarce resources are constantly present as in South Africa, it is appropriate to assess how resources designated for care of the newborn are used. Salaries for nursing personnel constitute a considerable percentage of the costs involved and while there are demands to reduce staff numbers, it must be acknowledged that adequate staffing levels are a pre-requisite for an acceptable standard of neonatal care.

The measurement of nursing workload or development of indicators of nursing intensity for ill newborns is problematical. The dependency scales which are used for adult patients are obviously of limited value in a situation where all patients are always $100 \%$ dependent. There are few tools for measuring neonatal nursing workload in the literature, and the nature of neonatal practice in a developing country could mean that such tools might need to be modified in the light of local conditions.

A preliminary exploration of possible ways of measuring nursing workload was undertaken in Groote Schuur Maternity Centre in the newborn care unit (MCN) in October/November 1993. This nursery operates primarily as a tertiary referral hospital for the Peninsula Maternal and Neonatal Service (PMNS). This is largely State funded and caters for approximately $80 \%$ of all births in the region. Roughly half of the annual 34000 deliveries in the PMNS take place in the community in the Midwife Obstetric Units (MOU). These mothers and babies are not considered to be risk for maternal or neonatal problems and both mother and baby are discharged home within six to eight hours of delivery. The remainder are booked to deliver at one of the secondary level hospitals in the region or if indicated, at the tertiary level referral Maternity Centre at Groote Schuur Hospital.

The policy regarding all well newborns in the PMNS is that as far as possible babies are not separated from their mothers. This means that newborns are not routinely adrritted to a nursery and accompany their mothers directly from the labour ward to the postnatal ward. This also applies to well babies who are delivered by caesarean section or forceps.

Only ill babies, low birth weight babies or babies requiring careful observation or frequent investigations are admitted to this nursery. MCN has an open visiting policy for the immediate family and siblings of all infants admitted. Mothers are encouraged to give as much care as they possibly can to their babies.

The majority of infants admitted here are preterm (approximately 90\%). However the absence of alternative community hospitals means that these babies have to remain in this hospital until fit for discharge home when they weigh around $2000 \mathrm{~g}$. In many other countries these infants would be transferred back to an institution nearer their homes as soon as their condition permitted.

The newborn care unit has an official total of 60 beds. This is divided into the following areas:

\section{Intensive Care Unit (ICU) (12 incubators)}

Infants here typically present with following problems - low birth weight $(<1000 \mathrm{~g})$, respiratory distress requiring assisted ventilation or levels of inspired oxygen above $35 \%$ or sophisticated monitoring.

\section{Nursery No. 2 (12 incubators)}

The population of this nursery has very uniform characteristics. As a rule they are all preterm, older than 48 hours and weigh between 1000 and $1500 \mathrm{gm}$. All are in incubators and are almost exclusively tube fed $12 \times 24$ hours.

\section{Nurseries $3 \& 4$ (20 cots and incubators)}

The population of this nursery is also relatively uniform. Babies weigh between 1500 and $1800 \mathrm{gm}$., they are either tube fod bottle fod or a combination of both. A baby who becomes ill or otherwise gives cause for concern is moved into another area of MCN. Mothers have free access to their babies and are encouraged to spend as much time as possible with their infants, breast feeding and helping with care.

Nursery 5 (10-12 cots)

This area is primarily for babies awaiting discharge and who require routine baby care.

Nursery 6 (Observation Nursery) (usually about 4 incubators and 4 cots)

The nursing workload in this area is strikingly varied. Infants admitted here weigh more than $1500 \mathrm{~g}$, are ill or unstable or require careful observation and frequent investigations. The length of stay in this area varies from a couple of hours to several weeks.

In addition there are 3 cubicles with space for one cot or incubator for isolation purposes when indicated.

\section{LITERATURE SURVEY}

"Ideally, a system of workload measurement should be suitable to the local situation and simultaneously facilitate comparability across wards/units, specialities, hospitals, districts and regions. At present such a system does not exist ... " (Arthur and James, 1994:562)

Much of the available literature describes methods of determining appropriate staffing levels and developing formulae to achieve this. Most of this is not applicable to neonates.

A report of the British Association for Perinatal Medicine in association with the Neonatal Nurses Association on categories of babies requiring neonatal care (1992:868-9) describes four categories of infants. The criteria however, relate more specifically only to seriously ill babies.

The second phase of the work done in the Liverpool Maternity Hospital and reported by Williams, Whelan, Weindling and Cooke (1993:534-8) describes 3 classes of infants in their quest to determine the nursing requirements of sick infants. Once the patients have been appropriately categorised and weighted a workload index can be calculated. Methods of determining the demand for nursing care are discussed with great clarity in the taxonomy of Arthur and 
James (1994: 558-565). The approach used in this study would be classed as using a "bottom-up" management approach. The strengths of this method can be its relative objectivity and simplicity in implementation but this must be balanced against the task-orientated approach and the fact that to implement a proper study is expensive.

A very useful conceptual framework was provided by the Patient Intensity for Nursing Index (PINI) as described by Prescott et al in 1991. This describes the four major constructs upon which nursing workload essentially depends. There are as follows:-

- severity of the illness

- intensity of the patient needs for nursing care

- the complexity of the nursing process, tasks and procedures

- time spent caring for a specific patient

It is interesting to relate each construct to the nursing workload in MCN.

\section{Severity of Illness}

This is essentially a medical construct, based on a medical diagnosis, and whilst it usually follows that the sicker the infant the greater the demand for nursing care, there are obvious exceptions. For example, the newborn with a cleft lip and palate may not be ill at all, but could need a great deal of nursing time and expertise to get feeding techniques established. Similarly the nursing effort expended to get a preterm infant suckling on the breast or to reassure and support an anxious parent need bear no relation to the "sickness" of the infant.

\section{Intensity of Needs for Nursing Care}

This involves the use of some sort of mechanism which uses patient characteristics to predict nursing requirements. Some systems for classification or categorisation of infants with a view to estimating nursing workload have recently been described in the literature. The one which appeared to be most relevant to local neonatal practice was that which originated in work commissioned by the Northern Regional Health Authority in the UK in 1982. The dependency scale which evolved, known as the Northern Neonatal Network (NNN) scale, (see Table 1) has been used to estimate staffing needs on the basis that infants can be categorised to give an indication of nursing care required. (NNN, Northern Regional Health Authority 1993:539).

\section{Complexity of Tasks}

This is a particularly important aspect of measurement of nursing work for hospital administrators since the level of staff employed to provide the skills directly relates to employment costs.

This is also a notoriously difficult aspect to measure particularly in the neonatal field. The simplest "task", eg. changing a napkin can, when performed by an observant and well-informed nurse, yield much valued information - renal and gastro-intestinal function, neurological status etc. Similarly, not all tasks are visible - how does one measure decision making? Newborn care is a very dynamic process and nurses are often required to initiate treatment procedures before medical staff are available for consultation. A recent and comprehensive analysis of nursing activities in the Mersey Regional Neonatal Intensive Care Unit is described by Williams et al (1993).

\section{Time Taken to Execute Tasks}

Subjecting an individual at work to scrutiny by an observer with a stop watch is not a popular exercise.

- The worker can feel threatened and uncomfortable; conversely the Hawthorne

\section{Table 1 NEONATAL NURSING DEPENDENCY SCALE AS CURRENTLY} USED BY THE NORTHERN NEONATAL NETWORK.

High dependency care

Low dependency care

A 1. Babies currently receiving respiratory support (endotracheal, nasal, or facemask IPPV, IMV, or CPAP)

B 2. Babies currently requiring $40 \%$ oxygen or more.

3. Babies whose whole fluid inlake was provided intravenously in the previous 24 hours.

5. Babies currently weighing less than $1000 \mathrm{gm}$.

C 6. Babies currently receiving some (but less than $40 \%$ ) oxygen.
4. Babies with a stoma or a pleural, peritoneal, or urethral drain in situ

7. Babies currently receiving some intravenous fluid.

8. Babies at least partially tube fed in the past 24 hours.

9. Babies who have had a fit or apnoec attack (>20 sec) in the previous 24 hours.

10. Babies currently weighing between $1000 \mathrm{gm}$ and $1750 \mathrm{gm}$.

Each baby is to be categorised by the first (that is the lowest numbered) defining condition IPPV: intermittent positive pressure ventilation; IMV: intermittent mandatory ventilation; CPAP. constant positive airway pressure. effect can be observed and work output can improve in quality and quantity.

- There are difficulties in nursing in deciding where a specific task begins and ends - it is not necessarily a discrete entity. .

- Nurses often perform a single task concurrently with others. Nursery nurses have been seen to be bottle feeding a baby, answering the telephone and copying laboratory results into the infants folder simultaneously.

- There is a marked reluctance to reduce nursing to a series of tasks which are measured by the time taken to execute the task without consideration of other qualitative factors.

\section{METHODOLOGY}

\section{Complexity of Tasks}

The nursing activities in the Mersey Regional Neonatal Intensive Care Unit as described by Williams et al (1993) was shown to several expert and experienced neonatal nurses to establish face validity for local nursing care. Certain activities were considered not applicable to the current care given in $\mathrm{MCN}$ (eg. collection of infant from dellvery suite or theatre, flying squad call) and were omitted from the list. Others were added (eg. including help with breast feeding under 9). Feeding, and Comforting as a specific entity was added under the heading Direct care. Team building activities were also added under the heading: Administration. The modified list follows:

\section{Direct Care}

1. Routine admission care

2. Subsequent "all care" (Weighing, washing, changing equipment, charts)

3. Physiotherapy and / or suctioning

4. Hygiene needs (nappy, mouth and cord care subsequent to 2 .)

5. Observations - nurse recording vital signs etc

6. Observations - (visual, in addition to 5.)

7. Drug administration

8. Care of intravenous infusion - changing fluid, re-siting etc

9. Feeding - includes help with breast feeding

10. Resuscitation

11. Specific tests eg. blood glucose

12. Collection of specimens eg. gastric aspirate, capillary blood sample

13. Care under phototherapy

14. Comforting infant

15. Escorting infant to another unit for investigations

16. Escorting infant for transfer to another ward

17. Care after the death of an infant

Indirect Care

18. Shift handover

19. Planning of nursing care

20. Discussion about management of 
workload/instructions to staff

21. Communication with and support of parents/family members

22. Communication with social worker

23. Ward rounds / discussion with medical staff

24. Assisting doctor / radiographer / ultrasonographer

25. Preparation for discharge home

26. Relocation of infant within unit

27. Changing equipment eg. ventilator circuits, puritan bottles

\section{Education}

28. Teach / demonstrate skills to parents

29. Teach / demonstrate skills to other staff

30. Counselling staff members

31. Assessing staff members

32. Attending meetings on unit (not if included as 18.)

33. Attending meetings off unit

34. New equipment demonstration

\section{Administration}

35. Duty rosters

36. Requests for repair, maintenance of equipment etc

37. Discussions with senior staff

38. Planning of social functions - Planning of team building activities

Support

39. Checking carts / ordering stores

40. Stocking up shelves etc

41. General tidying

42. Preparation for procedures/treatments/ tests

Telephone / Intercom

43. Answering Inquiries

44. Liaising with other departments

45. Liaising with other hospitals

46. Other

Personal

47. Meals etc

Domestic

48. Cleaning incubators

49. Other jobs normally done by domestics

Other

50. Delays (waiting for someone/something)

51. Available for task

This list gives an indication of the complexities of the job and the shortcomings of previous studies which have measured only direct care.

\section{Task Analysis}

A small convenience sample of experienced neonatally trained nurses was selected by virtue of the fact that they were on duty and in charge of Observation nursery on the day of observation. They were observed for periods ranging from four to six hours and activities were sampled at 5 minute intervals and then analysed to determine what percentage of time was spent in each dimension of care. Ethical considerations were addressed as described below.

\section{Intensity of Needs for Nursing}

The study was primarily a descriptive survey using nonparticipative observation to record the time spent on nursing interventions.

\section{Ethical Considerations}

The observer introduced herself on each occasion to the nurses involved and explained the purpose of the exercise. Nurses were informed that there would be no record of names and that the focus was on the duration of care given each infant and not the manner in which it was given. The nurse was given the opportunity to refuse to participate in this exercise.

Sampling:

A convenience sample from all categories of infants was randomly selected. The selection was influenced by factors such as the position of the incubator in the nursery - the observer wished to have a good vantage point but to be as unobtrusive as possible.

\section{Timing:}

This was continuous for each infant during the period of observation (2,5 - 4 hours). All nursing interventions were timed using the same nurses' watch with a second hand - this was done by a single observer. Care was timed with no regard to the level of nurse giving the care.

\section{Data collection:}

This included a brief perinatal history, age, weight, and any significant information, the time of commencement and completion of each nursing intervention as well as an abbreviated description of the intervention eg. nappy changing, observations, helping with $\mathrm{x}$-rays elc

\section{Analysis of Data:}

The total time devoted to each infant was divided by the duration of the observation period to obtain an hourly rate. From this an average hourly rate was calculated for each category of inf ant. These rates were compared with those determined in the NNN study.

\section{ASSUMPTIONS:}

- Certain activities in a neonatal unit take up $100 \%$ of the nurse's time during a specific period eg. admission of a baby to the ICU. Since this occurs only once in an average stay of 5-6 weeks this was not included in the observation period.

- Direct nursing care in a neonatal unit does not differ significantly between day and night.
FINDINGS \& DISCUSSION:

1. Complexity of Tasks:

TABLE 2: VARIETY OF NURSING TASKS

\begin{tabular}{|c|c|}
\hline $\begin{array}{l}\text { DIMENSION } \\
\text { OF CARE }\end{array}$ & $\begin{array}{r}\text { RANGE } \\
\text { OF TIME } \\
\text { SPENT }(\%)\end{array}$ \\
\hline Direct care & $15-30$ \\
\hline Indirect care & $13-27$ \\
\hline Education & $19-23$ \\
\hline Administration & $0 \cdot 3$ \\
\hline Support & $0-3$ \\
\hline Telephone & $1-19$ \\
\hline Personal & $15-16$ \\
\hline Domestic & 0.0 \\
\hline Other & $0-12$ \\
\hline \multicolumn{2}{|c|}{$\begin{array}{l}\text { Note: Low percentage of time spent on } \\
\text { administration is because one nurse is generally } \\
\text { responsible for this as it relates to the entire MCN }\end{array}$} \\
\hline \multicolumn{2}{|c|}{$\begin{array}{l}\text { Low percentage of time spent on support is a } \\
\text { favourable consequence of the "Cart system" of } \\
\text { centralised stocking and re-ordering of } \\
\text { consumable stores at GSH }\end{array}$} \\
\hline \multicolumn{2}{|c|}{$\begin{array}{l}\text { High percentage of time spent on personal can } \\
\text { be attrbuted to the four hour continuous } \\
\text { observation period oflen including a meal and or } \\
\text { lea break and is thus not an accurate reflection } \\
\text { laken over a } 12 \text { hour shim. }\end{array}$} \\
\hline
\end{tabular}

\section{Intensity of Needs:}

In addition to the difficulties generally associated with the timing of nursing tasks further problems arose in MCN. The "task" is often executed in a fragmented manner and by a multiplicity of nurses. One nurse will put the feeding tubes on top of the incubators, another will put out the bottles containing the feed; one will add the prescribed medication to the feed, another will actually administer the tube feed. The full recording of the feed in the notes might be done by consulting yet another nurse who bathed and changed the baby half an hour before feeding time.

This factory assembly line approach to care is worrying. It has evolved over a period of time marked by a steady erosion of staff numbers without a reduction in the number of infants requiring care. In terms of efficiency and economy of effort it can only be classed as a supert performance; however, for a variety of reasons this system of delivery of neonatal nursing care has inherent potential dangers. For the nurse it could mean a reduction in job satisf action and consequent "burn out"; for the infant, increased risk of cross-infection and lack of holistic care (see Table 3 ).

A similar process was followed for Categories "C" \& "D" infants in Nurseries 2 to 5.

"C" category infants have an average hourly rate of 6.5 minutes, with early morning $(07 \mathrm{~h} 00-10 \mathrm{~h} 00)$ rate significantly higher than afternoon and evening rates - $8.9: 4$ minutes per hour. This could be anticipated since the 


\section{TABLE 3: TIMES SPENT IN CARE GIVING}

\section{CATEGORY "A" (Intensive Care)}

1
2
3
4
5
6
7
8
9

Observation

Period in Mins.

150

180

180

180

150

150

180

180

180

9

Average rate per hour is 8 minutes

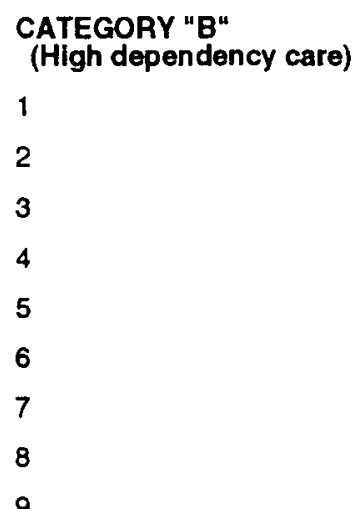

Average rate per hour is 11 minutes

Note: Intant 2 in " $B$ " category was a full term intant who presented with an unusual metabolic condition and required intense investigation. If this infant was excluded the average would be 8 minutes per hour.

infants are bathed and weighed in the mornings.

"D" category infants demand essentially the same amount of direct nursing care as " $\mathrm{C}$ " category, but in our practice there is a considerable amount of nursing time spent on indirect care and education such as liaising with social workers and ensuring parents can cope with the infant on discharge.

\section{Nursery 6 (Observation Nursery)}

Attempts to measure direct care were abandoned. The nursing workload was certainly heavier here than in other areas but since the purpose of the timing was primarily to verify the NNN scale of category of infant, it seemed that more value could be derived from an analysis of total workload. (See Table 2)

Factors contributing to the increased workload in this area:

1. The infants do not usually stay long in this area. This means that two of the most time-consuming nursing activities namely admission and discharge occur far more frequently here. A rough and not always complete record is kept of

$\begin{array}{rr}\begin{array}{c}\text { Total time } \\ \text { spent in mins. }\end{array} & \begin{array}{r}\text { Mean rate } \\ \text { of minutes } \\ \text { per hr. }\end{array} \\ 13 & 5.2 \\ 18 & 6.0 \\ 34 & 11.3 \\ 15 & 5.0 \\ 49 & 19.6 \\ 17 & 6.8 \\ 28 & 9.3 \\ 6 & 2.0 \\ 19 & 6.3\end{array}$

14.3

31.6

6.3

5.3

6.5

10.0

6.75

7.5

7.2
Departures

To mother in $\mathrm{LW} / \mathrm{W}$ ard

$\begin{array}{llll}5 \quad 10 & 15 & 20\end{array}$

To another hospital

eg.Transf to Red Cross

$\begin{array}{llll}3 & 5 & 10 & 20\end{array}$

Discharge home from MCN $10 \quad 15 \quad 2030$

Death - care arrangements $2030 \quad 35 \quad 45$

Transfer to

paediatric ward GSH $\quad 10152030$

2. Because the length of stay is relatively short, the population is a very dynamic one and nurses are constantly dealing with new babies and new parents. Notes and identification bands have to be frequently checked before any nursing intervention and this is time consuming.

3. The population in this nursery is often very diverse. Most babies fall into category B (high dependency care). There are also those who fall into categories $C$ and $D$ but who need skilled observation for the first 8 hours or so after birth. The lack of uniformity and variety of nursing demand makes the factory assembly line approach less likely to work in this area. Nurse/patient allocation is also problematic in an area where turnover is so rapid and unpredictable.

4. Despite the labile condition of many of these infants, medical help is not always readily available as in ICU. This requires well developed clinical skills and decision making ability on the part of the neonatal nurse.

Staffing - numbers:

NNN recommendations are as follows: enquiries concerning infants whereabouts. On some occasions there were as many as 24 admissions or discharges within 24 hours recorded.

An attempt to measure how much nursing time was devoted to these activities was made by asking the trained neonatal nurses who had considerable experience in this area to complete a simple questionnaire. The nurses were asked to ing what they considered was the average time (nursing time) spent on several different types of admissions and discharges. The times most consistently ringed were as follows:

Arrivals

Minutes

from elsewhere in MCN (eg ICU or 3 \& 4)

25710

from elsewhere in GSH

(eg ward, OT, MF)

$10 \quad 152025$

from outside

(eg MOU or other hospital) 15202530 low dependency: 4 babies per nurse

Categories A \& B

high dependency:

The current staff complement at MCN is: low dependency: 6-7 babies per nurse high dependency:

$3-4$ babies per nurse

Staffing - levels:

The increased workload of the registered nurse (RN) particularly in ICU is made possible by using carefully selected enrollet nursing assistants (ENAs) to support and help the RN This appears to be working extremely well in an atmosphere of mutual trust and respect. 2 babies per nurse 
Subjective accounts of what constituted excessive nursing workload were given by several RNs. The main factor mentioned related to instability of the infant's condition; others mentioned were quality of help available (neonatal experience was rated much more important than level of nurse). Quantity of staff was also important, but inexperienced "pairs of hands" were judged to be of little value in a crisis.

Limitations of the study - the exploratory nature which examined many aspects of care caused the scale of investigation to be small. The convenience sample makes findings not able to be generalised.

\section{Conclusion and Recommendations}

This study underlined the difficulties of measuring nursing workload in a neonatal setting. However, it would seem that classification of infants into two main groups i.e. high dependency care and low dependency care using the modified NNN scale is a better means of allocating members of nursing staff to babies than the conventional formula of nurses per cot. "It is babies, not cots, that generate work" (NNN 1993:543)

It should not be difficult or expensive to extend the activity sampling time period to 24 hours and expand the scope of the task analysis to include all levels of staff if the self-reporting method of William et al (1993: 535) was utilised. This would involve each nurse marking off on a prepared work card the time she spends on each activity. Since at any given time each nurse is caring for a single distinct group of infants (Observation nursery excluded) and the local hourly rate of direct care is known, it should be possible to calculate the rest from the work sheet.

Alternatively, all non-direct care can be based on cot occupancy, with only direct care being calculated by dependency classification described by Rhys Hearn (Goldstone 1980:238). Both of these methods merit a little more examination concerning their validity as a measurement of neonatal nursing workload in a tertiary level hospital in a developing country.

A perfect means of measurement will probably elude even the most dedicated researcher, but it is essential that in a situation with shrinking economic resources and clear demands for accountability that some means is devised to justify the pleas for more nurses. Pleas are made to hospital authorities by those responsible for the care of small vulnerable infants and who know the tragic consequences of too many sick babies and too few nurses.

\section{ACKNOWLEDGEMENTS}

Thanks to Mrs M Vermeulen and Professor A F Malan of Groote Schuur Matemity Centre for their encouragement and to all my Nursing colleagues who so patiently and good humouredly endured the scrutiny. Thanks also to Mrs A van der Walt, Deputy Director Nursing, Groote Schuur Hospital for permission to publish.

\section{REFERENCES}

Arthur T, James N. (1994): Determining nurse staffing levels: a critical review of the literature. Joumal of Advanced Nursing 19:558-565.

Goldstone L. (1980): Statistics in the Management of Nursing Services. Tunbridge Wells: Pitman Medical.

Northem Neonatal Network, Northem Regional Health Authority. Measuring neonatal nursing workload. Arch Dls Child (1993): 68:539-543.

Prescott P, Ryan JW, Soeker KL. Carstorr AH, Thompson KO, Phlllips CV. (1991): The Patient Intensity for Nursing Index: A Validity Assessment. Research in Nursing \& Health. $14 ; 214$.

Repon of working group of the British Association of Perinatal Medicine and Neonatal Nurses Association on categories of babies requining neonatal care. Arch Dis Child (1992):67:868-9.

Williams $\mathrm{S}$, Whelan A, Weindling AM, Cootze RWI. Nursing staff requirements for neonatal intensive care. Arch Dis Child (1993): 68:534-538.

\author{
JM van den Heever RM. \\ Groote Schuur Maternity Centre/ \\ Department of Nursing, \\ UNIVERSITY OF CAPE TOWN
}

